\title{
Florida Springs Land Use Information Tool ${ }^{1}$
}

\author{
Thomas Obreza ${ }^{2}$
}

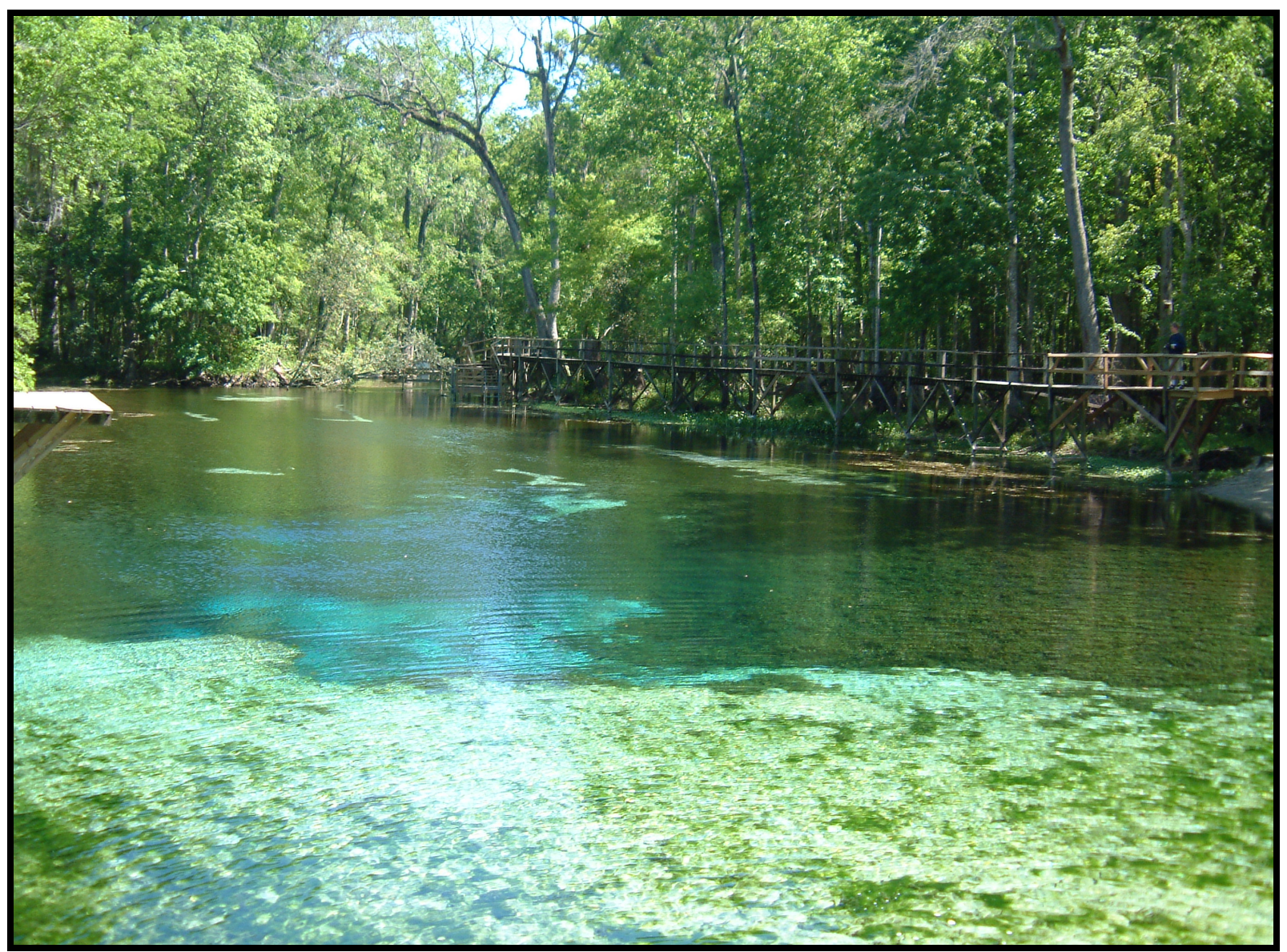

\section{Blue Springs, Gilchrist County, Florida \\ Photo taken by Greg Means, UF Soil and Water Science Dept.}

1. This document is CIR 1448, a circular of the Soil and Water Science Department, Florida Cooperative Extension Service, Institute of Food and Agricultural Sciences, University of Florida. Original publication date: April 2004. Visit the EDIS Web Site at http://edis.ifas.ufl.edu.

2. Thomas Obreza, Professor, Soil and Water Science Department, Florida Cooperative Extension Service, Institute of Food and Agricultural Sciences, University of Florida, Gainesville, 32611-0290. information and other services only to individuals and institutions that function without regard to race, color, sex, age, handicap, or national origin. For information on obtaining other extension publications, contact your county Cooperative Extension Service office. Florida Cooperative Extension Service/Institute of Food and Agricultural Sciences/University of Florida/Christine Taylor Waddill, Dean. 


\section{Florida Springs Land Use Information Tool}

\section{Table of Contents}

Introduction

Guidelines for Using the Information

Data Sources

Section 1: Nitrogen

Section 2a: Water Consumption - Agriculture and Horticulture

Section 2b: Groundwater Consumption - Residential

Section 3: Nitrogen Measured in Runoff

Section 4: Simulated Nitrogen Loading to Groundwater

Section 5: Simulated Effect of Best Management Practices (BMPs) on Nitrogen Loading to Groundwater

Section 6: Relative Pesticide Use

Section 7: Availability of Best Management Practices

Section 8: Glossary

Appendix: Land Uses Found Within the Florida Springs Study Area 


\section{Land Use Information Tool Introduction}

Purpose of this publication: To provide information about how land use influences the amount of nitrogen that may be imported as fertilizers or soil amendments, the amount of nitrogen that may be exported in the harvested portion of a crop, the amount of water that may be consumed, and the relative pesticide loading.

Intended audiences: State and local governments, land use planners, policymakers, consultants, land owners, and educators.

Scope: The information presented here applies to the "springs" area of north Florida. Florida's springs are classified by their average output (Table 1). Forty-one of Florida's 67 counties contain at least one $4^{\text {th }}$ magnitude or greater spring. First magnitude springs are found in 20 north and north-central counties (Fig. 1). The defined study area for this project was comprised of the 35 counties listed in Table 2 and illustrated in Fig. 2.

Table 1. Classification system for springs according to average discharge.

\begin{tabular}{ccc}
\hline Magnitude & \multicolumn{2}{c}{ Average flow } \\
\hline & cubic ft per second & million gallons per day \\
2 & 100 & 65 \\
3 & $10-100$ & $6.5-65$ \\
4 & $1-10$ & $0.65-6.5$ \\
& $<1$ & $<0.65$ \\
\hline
\end{tabular}

Table 2. Florida counties defining the project study area.

\begin{tabular}{lllll}
\hline Alachua & Dixie & Jackson & Madison & Suwannee \\
Bay & Gadsden & Jefferson & Marion & Taylor \\
Bradford & Gilchrist & Lake & Orange & Union \\
Calhoun & Gulf & Lafayette & Pasco & Volusia \\
Citrus & Hamilton & Leon & Putnam & Wakulla \\
Clay & Hernando & Levy & Seminole & Walton \\
Columbia & Holmes & Liberty & Sumter & Washington \\
\hline
\end{tabular}

Land uses within the study area were acquired using GIS coverages obtained from the Florida Geographic Data Library. Individual land use data files were generated by the Northwest, Suwannee River, and St. John's Water Management Districts. Most data were acquired between 1995 and 1999, with the Suwannee River Water Management District counties updated in 2002. A summary of land uses found is shown in the Appendix. 
- location of $1^{\text {st }}$ magnitude springs

Spring magnitudes contained within county boundaries

$1^{\text {st }}$ and lower

$2^{\text {nd }}$ and lower

$3^{\text {rd }}$ and lower

$4^{\text {th }}$ and lower

Fig. 1. Locations of Florida's springs.

Springs Project defined study area

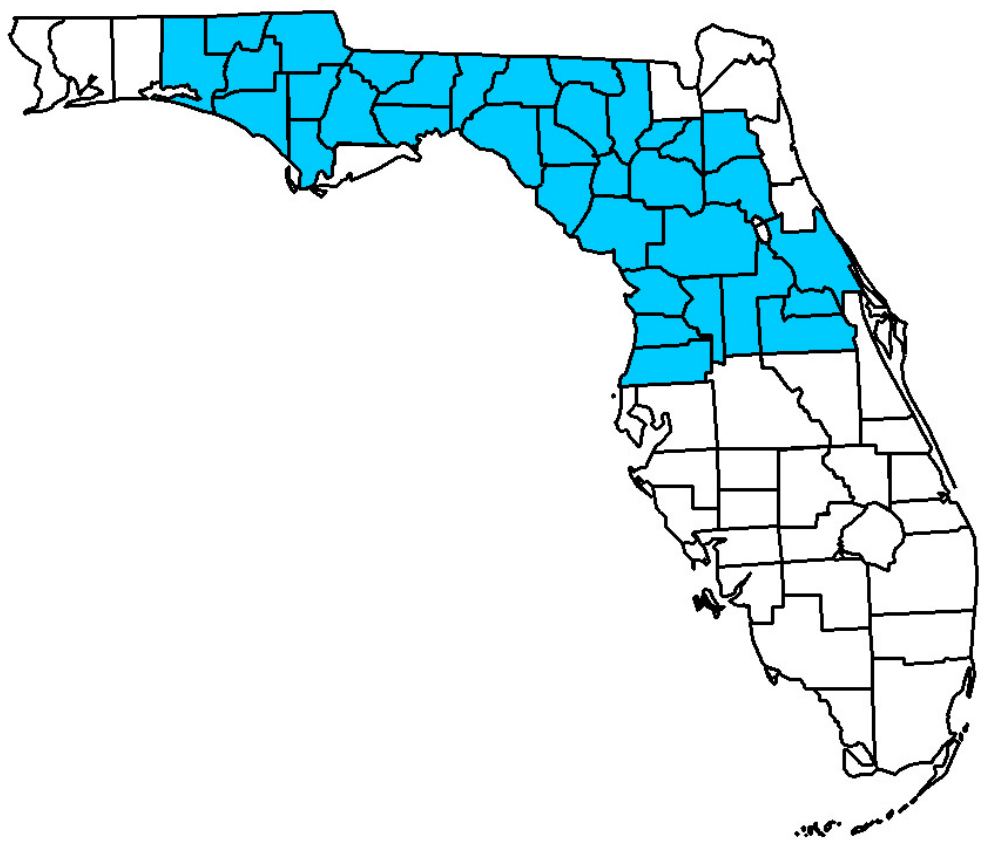

Fig. 2. The defined study area for this publication (35 counties). 


\section{Land Use Information Tool Guidelines for Using the Information}

\section{Please review these guidelines before using the information.}

\section{Intent of the Tool}

The intent of this tool is to provide information about how land use influences:

- The rate of nitrogen that may be applied to the land as fertilizers or soil amendments (nitrogen "imports").

- The rate or amount of nitrogen that may be removed from the land in the harvested portion of a crop (nitrogen "exports").

- The amount of water that may be consumed.

- The relative pesticide loading.

\section{Nitrogen movement to groundwater is site-specific (Sections 1 and 2)}

Knowing only nitrogen imports/exports and water consumption does not allow one to make valid comparisons between different land uses regarding contamination of groundwater by nitrate. Although these factors are important, they are only two components of nature's highly complex nitrogen cycle (Fig. 3).

What else can happen to nitrogen besides leaching to groundwater?

- Plants can absorb it.

- It can go off into the atmosphere as a gas.

- It can become part of the soil organic matter (humus).

- Soil microorganisms can use it.

Assuming that a site in question is within a springshed, other questions must be asked in order to use the information in this tool to help make decisions about it, such as:

- How much of the total land area receives nitrogen imports? For example, in residential areas only a portion of the lawns and landscapes may receive fertilizers.

- What level of management does the site receive? For example, "manicured" lawns and landscapes may receive high nitrogen fertilizer rates, while more "natural" landscapes may receive little or none.

- In the case of areas with septic tanks, what is the population density?

- Does the site receive supplemental irrigation? If yes, what type of irrigation system is used? Nitrogen fertilizer rates may differ depending on irrigation capacity.

- In the case of animal feeding operations, how many animals are there, and is manure exported off site or disposed of on site? If disposed of on site, how much area is available for land application?

\section{Use of Best Management Practices (Section 7)}

The key to preventing nitrogen from reaching groundwater lies with land use management. Where nitrogen imports are involved, best management practices (BMPs) have been developed that minimize the potential for groundwater contamination while maintaining economic viability. These BMPs were produced through the cooperative efforts of the Florida Dept. of Agriculture and Consumer Services, the Florida Dept. of Environmental Protection, the Univ of Florida, 
Florida's Water Management Districts, and the industries involved. Perhaps the most important function of this tool is to identify the many different land uses for which BMPs exist.

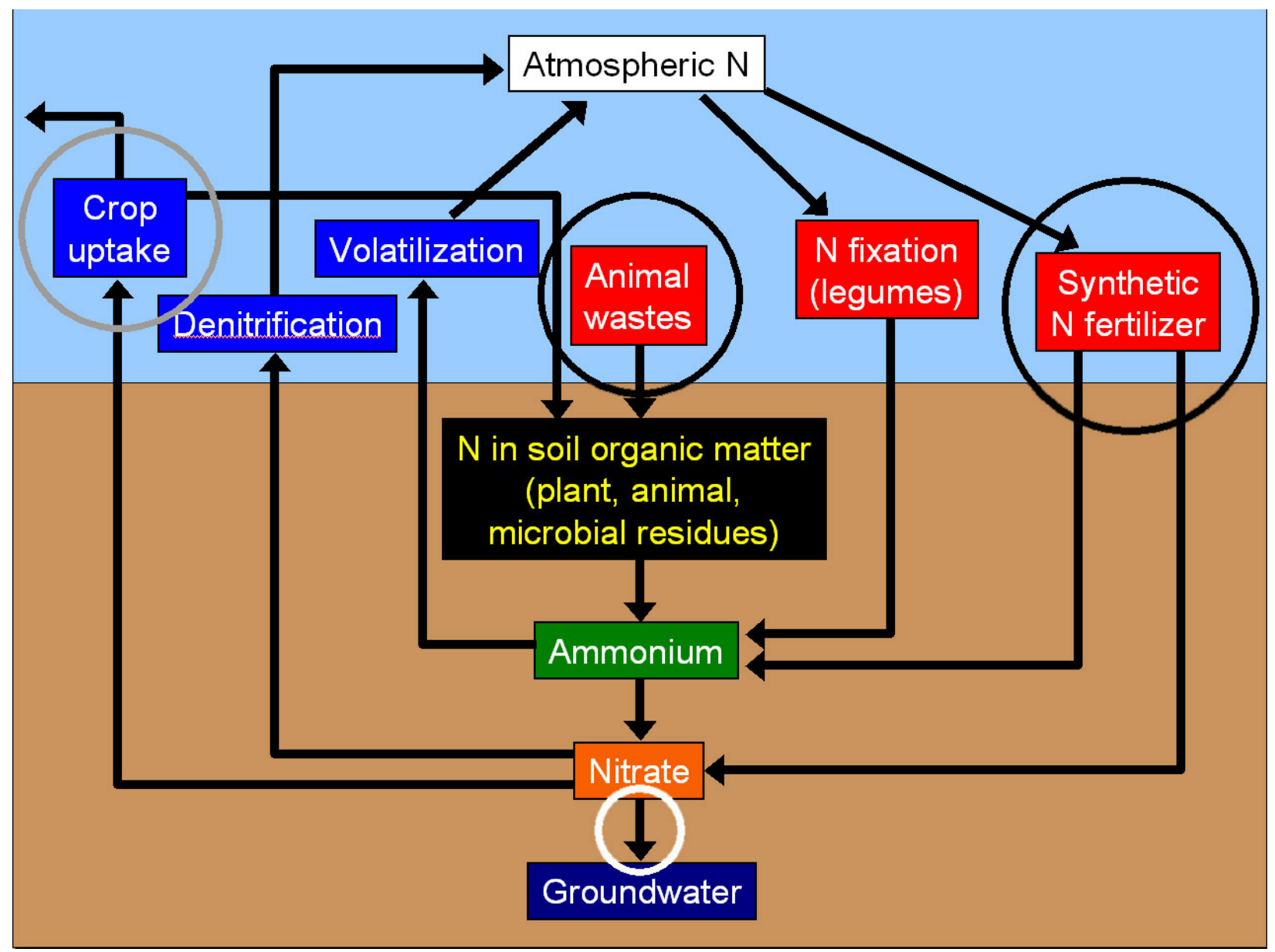

Fig. 3. A much-simplified version of the complex nitrogen cycle.

- $\quad \mathbf{N}$ imports as discussed in this tool are represented by the black circles.

- $\mathbf{N}$ export as discussed in this tool is represented by the grey circle.

- $\mathbf{N}$ leaching to groundwater is represented by the white circle. 


\section{Land Use Information Tool Data Sources}

Arnold, C.E. and T.E. Crocker. 1998. Pecan production in Florida. Horticultural Sciences Dept. Circular 280D. Univ. of Florida, Gainesville, FL.

Augustin, B.J. 2000. Water requirements for Florida turfgrasses. Dept. of Environmental Horticulture Bulletin 200. Univ. of Florida, Gainesville, FL.

Blevins, D., H., L. Allen, S. Colbett, and W. Gardner. 1996. Nutrition management for longleaf pinestraw. Woodland Owner Notes No. 30. North Carolina Cooperative Extension Service. North Carolina State Univ., Raleigh, NC.

Crocker, T. E. 1997. Alternative Opportunities for Small Farms: Muscadine Grape Production Review. Horticultural Sciences Dept. fact sheet RFAC 016 (EDIS DLN AC016), Univ. of Florida, Gainesville, FL

Florida Geographic Data Library, University of Florida Geoplan Center.

Florida Dept. of Transportation. 1999. Florida land use, cover and forms classification system handbook, $3^{\text {rd }}$ edition. Surveying and Mapping, Geographic Mapping Section, Florida Dept. of Transportation, Tallahassee, FL.

Harper, H. H. 1994. Stormwater loading rate parameters for central and south Florida. Environmental Research \& Design, Inc. Orlando, FL.

Hochmuth, G.L., and D.N. Maynard. 2000. Vegetable Production Guide for Florida. IFAS Publication SP 170. Univ. of Florida, Gainesville, FL.

Hochmuth, G.J., and E.A. Hanlon. 2000. IFAS standardized fertilization recommendations for vegetable crops. Horticultural Sciences Dept. Circular 1152. Univ. of Florida, Gainesville, FL.

Jokela, E.J., and A.L. Long. 2000. Using soils to guide fertilizer recommendations for southern pines. School of Forest Resources and Conservation Circular 1230. Univ. of Florida, Gainesville, FL.

Kidder, G., C.G. Chambliss, and R. Mylavarapu. 2001. UF/IFAS standardized fertilization recommendations for agronomic crops. Soil and Water Science Dept. Fact Sheet SL 129. Univ. of Florida, Gainesville, FL.

Knox, G., T. Broschat, G. Kidder, E. Gilman, L. Trenholm, R. Black, T. Wichman, D. Palmer, R. Zerba, C. White, A. Hunsberger, G. Israel, J. Cisar, K. Ruppert, D. Culbert, C. Kelly-Begazo and S.P. Brown. 2002. Fertilizer recommendations for landscape plants. Environmental Horticulture Dept. Publication ENH 858. Univ. of Florida, Gainesville, FL.

May, J.T. 1984. Nutrients and fertilization. In C. W. Lantz (ed.). Southern Pine Nursery Handbook. USDA Forest Service, Atlanta, GA. 
Sartain, J.B. 1998. Fertility considerations for sod production. Soil and Water Science Dept. Fact Sheet SL 52. Univ. of Florida, Gainesville, FL.

Sartain, J.B. 2000. General recommendations for fertilization of turfgrasses on Florida soils. Soil and Water Science Dept. Fact Sheet SL 21. Univ. of Florida, Gainesville, FL.

Sartain, J.B., and G.L. Miller. 2002. Recommendations for N, P, K and Mg for golf course and athletic field fertilization based on Mehlich 1 extractant. Soil and Water Science Dept. Fact Sheet SL 191. Univ. of Florida, Gainesville, FL.

Soil and Water Engineering Technology, Inc. (SWET). 1998. GIS watershed assessment final report. Part A - SR-WAM technical reference manual. Soil and Water Engineering Technology, Inc., Gainesville, FL.

United States Geological Survey, Tallahassee, FL.

Van Horn, H.H., D.R. Bray, R.A. Nordstedt, R.A. Bucklin, A.B. Bottcher, R.N. Gallaher, C.G. Chambliss, and G. Kidder. 1993. Water budgets for Florida dairy farms. Dairy Science Dept. Circular 1091. Univ. of Florida, Gainesville, FL.

Van Horn, H. H., G. L. Newton, R. A. Nordstedt, E. C. French, G. Kidder, D. A. Graetz, and C. G. Chambliss. 1998. Dairy manure management: Strategies for recycling nutrients to recover fertilizer value and avoid environmental pollution. Dairy and Poultry Sciences Dept. Circular 1016. Univ. of Florida, Gainesville, FL.

Whitty, E.B., and C.G. Chambliss. 2002. Water use and irrigation management of agronomic crops. Agronomy Dept. Publication SS-AGR-155. Univ. of Florida, Gainesville, FL.

Williamson, J. G., and P. Lyrene. Commercial blueberry production in Florida. IFAS Publication SP 179, Univ. of Florida, Gainesville, FL.

Williamson, J. G., and T.E. Crocker. 2000. Peaches and nectarines for Florida. Horticultural Sciences Dept. Circular 299D. Univ. of Florida, Gainesville, FL. 


\section{Land Use Information Tool \\ Section 1: Nitrogen \\ Imports and Exports}

\section{General comments}

1. Imports refer to nitrogen applied as commercial fertilizers, animal manures, biosolids, or wastewater.

2. Import rates are shown in lbs per acre on a treated area basis.

3. Exports refer to nitrogen removed from a site in the harvested portion of a crop.

\begin{tabular}{|c|c|c|}
\hline Land use & Nitrogen imports & Nitrogen exports \\
\hline \multicolumn{3}{|c|}{ Residential and Commercial } \\
\hline & Ibs $\mathrm{N}$ per acre per year & Ibs $N$ per acre per year \\
\hline $\begin{array}{l}\text { Residential, low and medium } \\
\text { density }\end{array}$ & $\begin{array}{c}\text { Lawns: } 0-220 \\
\text { Landscape Plants: } 0-264 \\
\text { Septic Tanks: } 5-14 \text { lbs N per person per year }\end{array}$ & 0 \\
\hline Residential, high density & \multirow{2}{*}{$\begin{array}{l}\text { Lawns: } 0-220 \\
\text { Landscape Plants: } 0-264\end{array}$} & \multirow{2}{*}{0} \\
\hline Commercial & & \\
\hline \multicolumn{3}{|c|}{$\begin{array}{l}\text { Comments on residential and commercial: } \\
\text { 1. Low density residential is less than one dwelling unit per acre. Medium density residential is one to five } \\
\text { dwelling units per acre. High density residential is greater than five dwelling units per acre. } \\
\text { 2. Nitrogen is imported primarily as fertilizer used in landscape maintenance, plus nitrogen discharged } \\
\text { from septic tanks if present. } \\
\text { 3. The range of nitrogen fertilization rates for lawns and landscapes represents low to high maintenance. }\end{array}$} \\
\hline \multicolumn{3}{|c|}{ Recreation and Golf Courses } \\
\hline & Ibs $N$ per acre per year & Ibs $N$ per acre per year \\
\hline $\begin{array}{l}\text { Parks and other recreation } \\
\text { areas }\end{array}$ & $\begin{array}{l}\text { Lawns and Landscapes: See Residential } \\
\text { Athletic Field: } \mathbf{8 7 - 2 2 0}\end{array}$ & 0 \\
\hline Golf Courses & $\begin{array}{c}\text { Greens: } 174-348 \\
\text { Tees: } 131-261 \\
\text { Fairways: } 174-218 \\
\text { Rough: } 87\end{array}$ & 0 \\
\hline
\end{tabular}




\begin{tabular}{|c|c|c|}
\hline Land use & Nitrogen imports & Nitrogen exports \\
\hline \multicolumn{3}{|c|}{ Pasture and Range } \\
\hline & Ibs $N$ per acre per year & See unit below \\
\hline Improved Perennial Grass & $120-160$ & $10-41$ lbs $N$ per ton hay \\
\hline Bahiagrass Pasture & $50-180$ & $8-27$ Ibs N per ton hay \\
\hline Unimproved Pasture & 0 & O lbs N per acre \\
\hline Native Range & $\mathbf{0}$ & 0 lbs $\mathrm{N}$ per acre \\
\hline \multicolumn{3}{|c|}{$\begin{array}{l}\text { Comments on pasture: } \\
\text { 1. Manure deposition by animals is not included in the nitrogen imports column. } \\
\text { 2. The range of nitrogen fertilization rates for pasture represents low to high maintenance. } \\
\text { 3. Hay yield must be known before a nitrogen export rate can be estimated. }\end{array}$} \\
\hline \multicolumn{3}{|c|}{ Field Crops } \\
\hline & Ibs $\mathrm{N}$ per acre per cropping season & See yield unit below \\
\hline Corn & 210 & $0.8 \mathrm{lbs} N$ per $56-\mathrm{Ib}$ bu \\
\hline Sorghum & 150 & $1.7 \mathrm{lbs} N$ per cwt \\
\hline Cotton & 60 & $\begin{array}{c}0.03 \text { lbs } N \text { per Ib of } \\
\text { seed+lint }\end{array}$ \\
\hline Wheat & 80 & $1.2 \mathrm{lbs} N$ per $60-\mathrm{Ib}$ bu \\
\hline Tobacco & 80 & $1.7 \mathrm{lbs} N$ per cwt \\
\hline Peanuts, Soybeans & 0 & $\begin{array}{c}\text { Peanuts: } 80 \text { lbs } \mathrm{N} \text { per ton } \\
\text { Soybeans: } 3.5 \mathrm{lbs} N \\
\text { per } \mathbf{6 0}-\mathrm{lb} \text { bu }\end{array}$ \\
\hline Tomato, potato, pepper & 200 & 4 lbs N per ton \\
\hline Beans & $60-100$ & $0.1 \mathrm{lbs} N$ per $28-\mathrm{lb}$ bu \\
\hline Cucumbers, Watermelons & 150 & 0.026 lbs N per cwt \\
\hline \multicolumn{3}{|c|}{$\begin{array}{l}\text { Comments on field crops: } \\
\text { 1. Cropping seasons usually range from } 3 \text { to } 6 \text { months. } \\
\text { 2. Nitrogen imports listed are the maximum recommended fertilizer rates. The actual rate applied by a } \\
\text { grower could be more or less. } \\
\text { 3. Crop yield must be known before nitrogen export can be estimated. } \\
\text { 4. Yield unit abbreviations: bu = bushel; cwt = hundredweight. }\end{array}$} \\
\hline \multicolumn{3}{|c|}{ Fruit Crops } \\
\hline & Ibs $N$ per acre per year & See yield unit below \\
\hline Citrus & $50-240$ & 0.13 lbs N per 90 -lb box \\
\hline Peaches & $80-100$ & $2.4 \mathrm{lbs} \mathrm{N}$ per ton \\
\hline \multicolumn{3}{|c|}{$\begin{array}{l}\text { Comments on fruit crops: } \\
\text { 1. Nitrogen imports listed are the maximum recommended fertilizer rates. The actual rate applied by a } \\
\text { grower could be more or less. } \\
\text { 2. Crop yield must be known before nitrogen export can be estimated. }\end{array}$} \\
\hline
\end{tabular}




\begin{tabular}{|c|c|c|}
\hline Land use & Nitrogen imports & Nitrogen exports \\
\hline \multicolumn{3}{|c|}{ Animal Feeding Operations } \\
\hline & See unit below & ----- \\
\hline Cattle Feedlots & $124 \mathrm{lbs} N$ per $1000 \mathrm{lb}$ animal per year & Unknown \\
\hline Dairy & $234-273$ lbs N per $1400 \mathrm{lb}$ cow per year & Unknown \\
\hline Laying Hens & $1.0 \mathrm{lbs} N$ per $4 \mathrm{lb}$ animal per year & Unknown \\
\hline Broiler Chickens & $0.9 \mathrm{lbs} N$ per $2 \mathrm{lb}$ animal per year & Unknown \\
\hline \multicolumn{3}{|c|}{$\begin{array}{l}\text { Comments on animal feeding operations: } \\
\text { 1. The imports in this section represent nitrogen in animal manure production. } \\
\text { 2. The number of animals must be known before the total quantity of manure nitrogen imported can be } \\
\text { estimated. } \\
\text { 3. More information about the feeding operation, particularly where the manure is being applied, must be } \\
\text { known before nitrogen export can be estimated. }\end{array}$} \\
\hline \multicolumn{3}{|c|}{ Horticulture } \\
\hline & Ibs $\mathrm{N}$ per acre per year & See unit below \\
\hline St. Augustine grass sod & 260 & Unknown \\
\hline Bahiagrass sod & 180 & Unknown \\
\hline Leatherleaf ferns & $100-350$ & $\begin{array}{l}52-164 \text { lbs N per } \\
\text { acre per year }\end{array}$ \\
\hline Vineyards & 100 & 2.2 lbs per ton \\
\hline \multicolumn{3}{|c|}{$\begin{array}{l}\text { Comments on horticulture: } \\
\text { 1. Nitrogen imports listed are the maximum recommended fertilizer rates. The actual rate applied by a } \\
\text { grower could be more or less. } \\
\text { 2. For vineyards, crop yield must be known before nitrogen export can be estimated. }\end{array}$} \\
\hline \multicolumn{3}{|c|}{ Forestry } \\
\hline & Ibs $\mathrm{N}$ per acre per growing cycle & See yield unit below \\
\hline Pine Tree Nursery & 200 & 125 Ibs N per acre \\
\hline Pine Tree Plantations & $\begin{array}{c}40-50 \text { (Young stands) } \\
150-200 \text { (Established stands) }\end{array}$ & $\begin{array}{l}\text { Whole tree harvesting: } \\
19 \text { lbs per } N \text { acre per year } \\
\text { Pine straw harvesting: } \\
2-6 \text { lbs } N \text { per } 1000 \text { lbs } \\
\text { of pine straw }\end{array}$ \\
\hline \multicolumn{3}{|c|}{$\begin{array}{l}\text { Comments on forestry: } \\
\text { 1. Established pine tree stands receive the above nitrogen imports once every } 6 \text { to } 8 \text { years. }\end{array}$} \\
\hline
\end{tabular}




\section{Land Use Information Tool \\ Section 2a: Water Consumption - Agriculture and Horticulture}

\section{General comments}

1. Water consumption is comprised of water obtained from a surface or groundwater source; it does not include rainfall.

2. Water consumption data (columns 2, 3, and 4) are from the year 2000, and represent volumes consumed for specific land uses within the Northwest Florida, Suwannee River, St. John's River, and Southwest Florida Water Management Districts.

3. If a wide range in water consumption for a particular land use is shown, it is likely due to a large difference in irrigation system efficiency, e.g. seepage irrigation vs. drip irrigation.

4. Typical water use (right-hand column) is the projected amount of irrigation needed for an average growing season.

\begin{tabular}{|c|c|c|c|c|}
\hline \multirow[b]{2}{*}{ Land Use } & \multicolumn{3}{|c|}{ Water consumption } & \multirow{2}{*}{$\begin{array}{c}\text { Typical } \\
\text { water use }\end{array}$} \\
\hline & $\begin{array}{l}\text { Lowest } \\
\text { observed }\end{array}$ & $\begin{array}{c}\text { Highest } \\
\text { observed }\end{array}$ & Average & \\
\hline & \multicolumn{3}{|c|}{ gallons per acre per day } & \\
\hline Soybeans & 423 & 677 & 550 & $7 \mathrm{in} /$ season \\
\hline Sorghum & 585 & 621 & 603 & $6 \mathrm{in} / \mathrm{season}$ \\
\hline Cotton & 521 & 1156 & 839 & \\
\hline Peanuts & 602 & 1405 & 947 & $7 \mathrm{in} /$ season \\
\hline Corn & 803 & 1685 & 1135 & $12 \mathrm{in} /$ season \\
\hline Tobacco & 1065 & 1308 & 1187 & $7 \mathrm{in} /$ season \\
\hline Wheat & 1029 & 1420 & 1225 & \\
\hline All field crops & 584 & 1261 & 946 & \\
\hline Watermelon & 649 & 1919 & 1104 & $10 \mathrm{in} /$ season \\
\hline Tomato & 757 & 4574 & 2666 & $10 \mathrm{in} /$ season \\
\hline All vegetables & 668 & 2893 & 1463 & \\
\hline Peaches & 2000 & 2000 & 2000 & \\
\hline Citrus & 1349 & 3515 & 2415 & $15-20$ in/year \\
\hline All fruit crops & 977 & 3505 & 1710 & \\
\hline & & & & \\
\hline Pasture hay & 730 & 1834 & 1309 & \\
\hline Field-grown ornamentals & 1298 & 2965 & 2230 & \\
\hline Sod & 1002 & 4675 & 2474 & \\
\hline Container-grown ornamentals & 2299 & 9638 & 4912 & \\
\hline Greenhouse-grown ornamentals & 2143 & 9444 & 5794 & \\
\hline All ornamentals/grasses & 1386 & 2479 & 1875 & \\
\hline Other grass and landscape & 1312 & 1996 & 1654 & \\
\hline Golf courses & 1845 & 4374 & 2506 & \\
\hline All golf course and landscape & 1486 & 2575 & 1916 & \\
\hline Dairy & & & $175-400 \mathrm{~g}$ & r animal per da \\
\hline
\end{tabular}




\section{Land Use Information Tool \\ Section 2b: Groundwater Consumption - Residential}

\section{General comments}

1. Water consumption is comprised of water obtained from groundwater sources only.

2. Data were reported by the US Geological Survey in January, 2003.

\begin{tabular}{|c|c|c|c|c|c|c|c|c|}
\hline \multirow{4}{*}{ County } & \multirow{2}{*}{\multicolumn{3}{|c|}{ Population }} & \multicolumn{4}{|c|}{ Groundwater use } & \multirow{3}{*}{$\begin{array}{c}\text { Total } \\
\text { groundwater } \\
\text { use }\end{array}$} \\
\hline & & & & \multicolumn{2}{|c|}{ Total used } & \multicolumn{2}{|c|}{ Per capita } & \\
\hline & \multirow[t]{2}{*}{ Total } & \multirow[t]{2}{*}{$\begin{array}{l}\text { Public } \\
\text { supply }\end{array}$} & \multirow[t]{2}{*}{$\begin{array}{c}\text { Self } \\
\text { supply }\end{array}$} & $\begin{array}{l}\text { Public } \\
\text { supply }\end{array}$ & $\begin{array}{c}\text { Self } \\
\text { supply }\end{array}$ & $\begin{array}{l}\text { Public } \\
\text { supply }\end{array}$ & $\begin{array}{c}\text { Self } \\
\text { supply }\end{array}$ & \\
\hline & & & & \multicolumn{2}{|c|}{ million gal/day } & \multicolumn{2}{|c|}{ gal/day } & mgd \\
\hline Alachua & 217,955 & 179,118 & 38,837 & 28.26 & 4.12 & 158 & 106 & 32.38 \\
\hline Bay & 148,217 & 129,300 & 18,917 & 6.28 & 2.01 & 49 & 106 & 8.29 \\
\hline Bradford & 26,088 & 8,338 & 17,750 & 1.38 & 1.89 & 166 & 106 & 3.27 \\
\hline Calhoun & 13,017 & 4,224 & 8,793 & 0.75 & 0.93 & 178 & 106 & 1.68 \\
\hline Citrus & 118,085 & 66,234 & 51,851 & 13.97 & 7.20 & 211 & 139 & 21.17 \\
\hline Clay & 140,814 & 100,785 & 40,029 & 14.77 & 4.24 & 147 & 106 & 19.01 \\
\hline Columbia & 56,513 & 21,235 & 35,278 & 3.67 & 3.74 & 173 & 106 & 7.41 \\
\hline Dixie & 13,827 & 4,622 & 9,205 & 0.67 & 0.98 & 145 & 106 & 1.65 \\
\hline Gadsden & 45,087 & 27,632 & 17,455 & 3.06 & 1.85 & 111 & 106 & 4.91 \\
\hline Gilchrist & 14,437 & 1,850 & 12,587 & 0.27 & 1.33 & 146 & 106 & 1.60 \\
\hline Gulf & 13,332 & 10,338 & 2,994 & 1.47 & 0.32 & 142 & 107 & 1.79 \\
\hline Hamilton & 13,327 & 6,366 & 6,961 & 0.95 & 0.74 & 149 & 106 & 1.69 \\
\hline Hernando & 130,802 & 116,025 & 14,777 & 20.26 & 1.41 & 175 & 95 & 21.67 \\
\hline Holmes & 18,564 & 5,860 & 12,704 & 1.38 & 1.35 & 235 & 106 & 2.73 \\
\hline Jackson & 46,755 & 16,348 & 30,407 & 2.46 & 3.22 & 150 & 106 & 5.68 \\
\hline Jefferson & 12,902 & 5,010 & 7,892 & 0.72 & 0.84 & 144 & 106 & 1.56 \\
\hline Lafayette & 7,022 & 1,264 & 5,758 & 0.20 & 0.61 & 158 & 106 & 0.81 \\
\hline Lake & 210,528 & 171,137 & 39,391 & 39.92 & 4.29 & 233 & 109 & 44.21 \\
\hline Leon & 239,452 & 198,937 & 40,515 & 35.70 & 4.29 & 179 & 106 & 39.99 \\
\hline Levy & 34,450 & 11,066 & 23,384 & 2.16 & 3.95 & 195 & 169 & 6.11 \\
\hline Liberty & 7,021 & 2,764 & 4,257 & 0.39 & 0.45 & 141 & 106 & 0.84 \\
\hline Madison & 18,733 & 7,166 & 11,567 & 1.65 & 1.23 & 230 & 106 & 2.88 \\
\hline Marion & 258,916 & 136,842 & 122,074 & 27.99 & 16.42 & 205 & 135 & 44.41 \\
\hline Orange & 896,344 & 813,152 & 83,192 & 186.15 & 8.82 & 229 & 106 & 194.97 \\
\hline Pasco & 344,765 & 275,800 & 68,965 & 35.23 & 4.50 & 128 & 65 & 39.73 \\
\hline Putnam & 70,423 & 23,311 & 47,112 & 3.20 & 4.99 & 137 & 106 & 8.19 \\
\hline Seminole & 365,196 & 339,403 & 25,793 & 66.90 & 2.73 & 197 & 106 & 69.63 \\
\hline Sumter & 53,345 & 28,243 & 25,102 & 4.44 & 4.57 & 157 & 182 & 9.01 \\
\hline Suwannee & 34,844 & 9,393 & 25,451 & 1.40 & 2.70 & 149 & 106 & 4.10 \\
\hline Taylor & 19,256 & 10,289 & 8,967 & 1.73 & 0.95 & 168 & 106 & 2.68 \\
\hline Union & 13,442 & 3,155 & 10,287 & 0.36 & 1.10 & 114 & 107 & 1.46 \\
\hline Volusia & 443,343 & 414,851 & 28,492 & 54.90 & 3.02 & 132 & 106 & 57.92 \\
\hline Wakulla & 22,863 & 9,285 & 13,578 & 2.19 & 1.44 & 236 & 106 & 3.63 \\
\hline Walton & 40,601 & 39,024 & 1,577 & 7.35 & 0.17 & 188 & 108 & 7.52 \\
\hline Washington & 20,973 & 7,565 & 13,408 & 1.16 & 1.42 & 153 & 106 & 2.58 \\
\hline Totals & $4,131,239$ & $3,205,932$ & 925,307 & 573.34 & 103.82 & & & 677.16 \\
\hline Average & & & & & & 166 & 110 & \\
\hline
\end{tabular}




\section{Land Use Information Tool Section 3: Nitrogen Measured in Runoff}

\section{Comment}

In the 1990s, Environmental Research \& Design, Inc. of Orlando compiled results of an extensive literature search and analysis of measured nitrogen loading rates during runoff events from various land uses in central and south Florida (Harper, 1994). The values in this table represent runoff nitrogen (i.e. surface water movement to streams and lakes), not leached nitrogen. However, nitrogen loss from different land uses can still be compared. Note that land uses with greater amounts of impervious surfaces lose more nitrogen to runoff than land uses where water infiltration dominates.

\begin{tabular}{|l|c|}
\hline \multicolumn{1}{|c|}{ Land use } & \multicolumn{1}{|c|}{$\begin{array}{c}\text { Mass loading of total N } \\
\text { lbs per acre per year }\end{array}$} \\
\hline Recreation/Open Space & 2.4 \\
\hline Wetlands & 4.0 \\
\hline Mining/Extractive & 4.9 \\
\hline Agriculture - Row Crops & 6.2 \\
\hline Residential, Low Density & 6.4 \\
\hline Agriculture - Citrus & 6.4 \\
\hline Open Water/Lakes & 7.1 \\
\hline Agriculture - General & 7.9 \\
\hline Agriculture - Pasture & 9.9 \\
\hline Residential, Single Family & 10.4 \\
\hline Commercial, Low-Intensity & 11.5 \\
\hline Highway & 14.8 \\
\hline Industrial & 16.1 \\
\hline Residential, Multi-Family & 18.8 \\
\hline Commercial, High Intensity & 28.7 \\
\hline
\end{tabular}




\section{Land Use Information Tool Section 4: Simulated Nitrogen Loading to Groundwater}

\section{Comment}

In the late 1990s, Soil and Water Engineering Technology, Inc. performed a watershed assessment with respect to water quality for the Suwannee River Water Management District (SWET, 1998). They used mathematical modeling to simulate the relative impacts of different land uses on nitrogen loading to groundwater. The values in this table should be interpreted with caution since they do not represent measured values. However, it is easy to see that some land uses have more of a predicted impact than others.

\begin{tabular}{|l|c|}
\hline \multicolumn{1}{|c|}{ Land Use } & $\begin{array}{c}\text { Nitrogen Loading to Groundwater } \\
\text { lbs per acre per year }\end{array}$ \\
\hline Managed landscapes & 11 \\
\hline Agriculture - Sod farm & 16 \\
\hline Residential, low density & 19 \\
\hline Agriculture - Peach orchard & 36 \\
\hline Agriculture - Pecan orchard & 36 \\
\hline Agriculture - Row crops & 38 \\
\hline Agriculture - Poultry feeding operation & 43 \\
\hline Horse farm & 46 \\
\hline Agriculture - Blueberries & 54 \\
\hline Agriculture - Dairy & 62 \\
\hline Animal race tracks & 64 \\
\hline Residential, medium density & 66 \\
\hline Nursery - Trees & 157 \\
\hline Zoo & 158 \\
\hline Nursery - Ornamentals & 188 \\
\hline
\end{tabular}




\section{Land Use Information Tool Section 5: Simulated Effect of Best Management Practices (BMPs) on Nitrogen Loading to Groundwater}

\section{Comment}

In this exercise, SWET used their simulation model to predict nitrogen loading to groundwater for several land uses in their current condition, and then re-ran the simulation after implementing recommended BMPs (SWET, 1998). The model predicted that by implementing BMPs, the nitrogen loading would be reduced from 17 to $72 \%$ compared with the current condition.

\begin{tabular}{|c|c|c|c|}
\hline \multirow{3}{*}{ Land Use } & \multicolumn{3}{|c|}{ Nitrogen Loading to Groundwater } \\
\hline & $\begin{array}{c}\text { Existing } \\
\text { condition }\end{array}$ & $\begin{array}{c}\text { After } \\
\text { implementation } \\
\text { of BMPs }\end{array}$ & $\begin{array}{l}\text { Reduction due } \\
\text { to BMP } \\
\text { implementation }\end{array}$ \\
\hline & \multicolumn{2}{|c|}{ Ibs per acre per year } & $\%$ \\
\hline Horse farms & 39 & 11 & 72 \\
\hline Agriculture - Peach and pecan orchards & 42 & 29 & 31 \\
\hline Agriculture - Row crops & 46 & 19 & 59 \\
\hline Agriculture - Poultry feeding operations & 46 & 20 & 57 \\
\hline Agriculture - Blueberries & 59 & 40 & 32 \\
\hline Agriculture - Dairy & 62 & 29 & 53 \\
\hline Residential - Medium density & 66 & 55 & 17 \\
\hline Nursery - Ornamentals & 200 & 131 & 35 \\
\hline
\end{tabular}




\title{
Land Use Information Tool Section 6: Relative Pesticide Use
}

\author{
Pesticides include insecticides, miticides, herbicides, and fungicides.
}

\section{Comment}

Pesticides are expensive, so they are used sparingly where the value of the crop or plant production is low. They tend to be applied more where crop value is high or visual aesthetics are important. In general, the more highly-valued the product or plant, the more likely that pesticides will be used.

\section{Rating Scale}

None: Pesticides not likely used.

Low: Pesticides applied less than once per month.

Medium: Pesticides applied one to four times per month.

High: Pesticides applied more than once per week.

\begin{tabular}{|l|c|l|}
\hline \multicolumn{1}{|c|}{ Land Use } & $\begin{array}{c}\text { Relative } \\
\text { Pesticide } \\
\text { Use }\end{array}$ & \multicolumn{1}{|c|}{ Comments } \\
\hline Residential and Commercial & $\begin{array}{c}\text { None to } \\
\text { Medium }\end{array}$ & $\begin{array}{l}\text { Pesticide application depends on maintenance } \\
\text { level desired by landscape manager. }\end{array}$ \\
\hline Parks and Recreation & $\begin{array}{c}\text { None to } \\
\text { Medium }\end{array}$ & $\begin{array}{l}\text { Pesticides more likely to be applied to high } \\
\text { maintenance turf situations like athletic fields. }\end{array}$ \\
\hline Golf Courses & Medium & $\begin{array}{l}\text { As the quality of a golf course increases, the } \\
\text { amount of pesticides applied tends to increase. }\end{array}$ \\
\hline Pasture and Range & $\begin{array}{c}\text { None to } \\
\text { Low }\end{array}$ & $\begin{array}{l}\text { Difficult to justify pesticide applications to low } \\
\text { value crops. }\end{array}$ \\
\hline Field Crops & $\begin{array}{c}\text { Low to } \\
\text { Medium }\end{array}$ & $\begin{array}{l}\text { Pesticide application depends on crop value and } \\
\text { pest pressure. }\end{array}$ \\
\hline Fruit Crops & Low & $\begin{array}{l}\text { Pesticide application depends on crop value and } \\
\text { pest pressure. }\end{array}$ \\
\hline Animal Feeding Operations & $\begin{array}{c}\text { None to } \\
\text { Low }\end{array}$ & \\
\hline Horticulture & $\begin{array}{c}\text { Medium to } \\
\text { High }\end{array}$ & $\begin{array}{l}\text { Pesticide application depends on crop value and } \\
\text { pest pressure. }\end{array}$ \\
\hline Forestry & $\begin{array}{c}\text { None to } \\
\text { Low }\end{array}$ & $\begin{array}{l}\text { Difficult to justify pesticide applications to low } \\
\text { value crops. }\end{array}$ \\
\hline
\end{tabular}




\section{Land Use Information Tool Section 7: Availability of Best Management Practices (BMPs)}

\section{Comment}

BMPs are available for most of the land uses found in the Florida Springs project study area.

\begin{tabular}{|c|c|}
\hline Land Use & Applicable BMP manual or publication \\
\hline $\begin{array}{l}\text { Residential } \\
\text { and } \\
\text { Commercial }\end{array}$ & $\begin{array}{l}\text { Florida Dept. of Environmental Protection. 2002. Florida Green Industries Best } \\
\text { Management Practices for Protection of Water Resources in Florida. Florida Dept. of } \\
\text { Environmental Protection, Tallahassee, FL. }\end{array}$ \\
\hline $\begin{array}{l}\text { Parks and } \\
\text { Recreation }\end{array}$ & $\begin{array}{l}\text { Florida Dept. of Environmental Protection. 2002. Florida Green Industries Best } \\
\text { Management Practices for Protection of Water Resources in Florida. Florida Dept. of } \\
\text { Environmental Protection, Tallahassee, FL. }\end{array}$ \\
\hline Golf Courses & $\begin{array}{l}\text { Elliott, M. L., and J. B. Unruh. 1998. Best Management Practices for Florida Golf } \\
\text { Courses. Institute of Food and Agricultural Sciences, Univ. of Florida, Gainesville, FL. }\end{array}$ \\
\hline $\begin{array}{l}\text { Pasture and } \\
\text { Range }\end{array}$ & $\begin{array}{l}\text { Florida Cattlemen's Association. 1999. Water Quality Best Management Practices for } \\
\text { Cow/Calf Operations in Florida. Florida Cattlemen's Association, Kissimmee, FL. }\end{array}$ \\
\hline Field Crops & $\begin{array}{l}\text { Florida Dept. of Agriculture and Consumer Services, Office of Agricultural Water Policy. } \\
\text { 2003. Florida Vegetable and Agronomic Crop Water Quality and Quantity Best } \\
\text { Management Practices Manual. Florida Dept. of Agriculture and Consumer Services, } \\
\text { Tallahassee, FL. (In development.) }\end{array}$ \\
\hline Fruit Crops & $\begin{array}{l}\text { Florida Dept. of Agriculture and Consumer Services, Office of Agricultural Water Policy. } \\
\text { 2002. Nitrogen Best Management Practices (BMPs) for Florida Citrus. Florida Dept. of } \\
\text { Agriculture and Consumer Services, Tallahassee, FL. }\end{array}$ \\
\hline $\begin{array}{l}\text { Animal } \\
\text { Feeding } \\
\text { Operations }\end{array}$ & $\begin{array}{l}\text { Van Horn, H. H., G. L. Newton, R. A. Nordstedt, E. C. French, G. Kidder, D. A. Graetz, and } \\
\text { C. G. Chambliss. 1998. Dairy manure management: Strategies for recycling nutrients } \\
\text { to recover fertilizer value and avoid environmental pollution. Dairy and Poultry } \\
\text { Sciences Dept. Circular 1016. Univ. of Florida, Gainesville, FL. }\end{array}$ \\
\hline Horticulture & $\begin{array}{l}\text { Stamps, R. H. 1995. Irrigation and Nutrient Management Practices for Commercial } \\
\text { Leatherleaf Fern Production in Florida. Univ. of Florida, IFAS, Gainesville, FL. } \\
\text { Florida Dept. of Agriculture and Consumer Services, Office of Agricultural Water Policy. } \\
\text { 2003. Interim Measure for Florida Producers of Container-Grown Plants. Florida Dept. } \\
\text { of Agriculture and Consumer Services, Tallahassee, FL. }\end{array}$ \\
\hline Forestry & $\begin{array}{l}\text { Florida Dept. of Agriculture and Consumer Services, Division of Forestry. } 2000 . \\
\text { Silviculture Best Management Practices. Florida Dept. of Agriculture and Consumer } \\
\text { Services, Tallahassee, FL. }\end{array}$ \\
\hline
\end{tabular}




\section{Land Use Information Tool Section 8: Glossary}

- Abbreviations

○ bu - bushel.

○ cwt - hundredweight (a 100-lb unit).

- Animal Waste - Manure and urine produced by farm animals.

- Crop Uptake - Nutrients taken up from the soil by roots and incorporated into plant tissues.

- Denitrification - Biological conversion of soil nitrate $\left(\mathrm{NO}_{3}{ }^{-}\right)$to gaseous forms of $\mathrm{N}$. This reaction occurs only in the absence of oxygen.

- Fertilizer - Any substance containing one or more recognized plant nutrients that is applied for its plant nutrient content.

\section{- Land Use Categories}

- Low-Density Residential - A rural area with lot sizes greater than 1 acre or less than one dwelling unit per acre. Another term for this category is "Rural Residential."

- Medium-Density Residential - A density of one to five dwellings per acre. Another term for this category is "Suburban Residential."

- High-Density Residential - A density of greater than five dwellings per acre. Another term for this category is "Urban Residential."

- Single-Family Residential - Typical detached home community with lot sizes generally less than 1 acre and dwelling densities greater than one dwelling unit per acre; duplexes constructed on onethird to one-half acre lots are also included in this category.

- Multi-Family Residential - Residential land use consisting primarily of apartments, condominiums, and cluster homes.

- Low-Intensity Commercial - Areas that receive only a moderate amount of traffic volume in areas where cars are parked during the day for extended periods of time. These areas include universities, schools, professional office sites, and small shopping centers.

- High-Intensity Commercial - Land use consisting of commercial areas with high traffic volume with constant traffic moving in and out of the area. These areas include downtown areas, commercial office sites, regional malls, and associated parking lots.

- Industrial - Land uses include manufacturing, shipping and transportation services, sewage treatment facilities, water supply plants, and solid waste disposal. 
- Highway - Includes major road systems such as interstate highways and major arteries and thoroughfares. Roadway areas associated with residential, commercial, and industrial land uses are included with those particular categories.

- Agriculture - Activities include animal production, grazing, row crops, citrus, and related activities.

- Recreation/Open Space - Includes recreational land such as parks and ball fields, open space, barren land, undeveloped land that may be occupied by native vegetation, rangeland, and power lines. This land does not include golf course areas that are heavily fertilized and managed; golf course areas have runoff characteristics similar to single-family residential areas.

- Mining/Extractive - A wide variety of mining activities for resources like phosphate, sand, gravel, clay, and shell.

- Wetlands - A wide range of diverse wetland types such as hardwood wetlands, cypress stands, grassed wetlands, freshwater marsh, and mixed wetland associations.

○ Open Water/Lakes - Open water and lakes, rivers, reservoirs, and other open water bodies.

- Nitrogen Export - Nitrogen that may be removed from the land in the harvested portion of a crop.

- Nitrogen Fixation - Biological conversion of atmospheric $\mathrm{N}_{2}$ gas to plant-available $\mathrm{N}$ by Rhizobia associated with the root system of leguminous plants.

- Nitrogen Import - Nitrogen that may be applied to the land as fertilizers or soil amendments.

- Pesticides - Includes herbicides (weed killers), insecticides (bug killers), nematicides, and fungicides.

- Soil Amendment - A material applied to improve or enhance soil characteristics for plant growth. A soil amendment may also contain required plant nutrients.

- Volatilization - Conversion of ammonium $\left(\mathrm{NH}_{4}{ }^{+}\right)$from manure, fertilizer, or the soil to gaseous ammonia $\left(\mathrm{NH}_{3}\right)$, which enters the atmosphere. 


\section{Appendix}

Land uses found within the Florida springs study area.

\begin{tabular}{|c|c|c|c|}
\hline \multicolumn{4}{|c|}{ Urban and Built-Up } \\
\hline Residential, Low Density & Oil and Gas Storage & Other Light Industry & Governmental \\
\hline Ranchettes Fixed (>5 AC/DU) & Mixed Commercial and Services & Plastic Pipe Plant & Correctional \\
\hline Low Density Residential Mobile & Cemeteries & Cement Plant & Municipal Prison \\
\hline Mobile Home Units & Commercial Under Construction & Chemical Processing & State Prison \\
\hline Ranchettes Mobile & Industrial & Other Heavy Industrial & Other Institutional Facility \\
\hline Low Density Residential Mixed & Food Processing & Pre-Stressed Concrete Plants & Institutional under Construction \\
\hline Ranchettes Mixed & Grain and Legume Processing & Extractive & Recreational \\
\hline Residential, Medium Density & Meat Packing Facility & Heavy Mineral Mine & Swimming Beach \\
\hline Mobile Home Units, Medium Density & Poultry and/or Egg Processing & Peat & Golf Courses \\
\hline Medium Density Residential Mixed & Seafood Processing & Strip Mines & Automobile Racing Track \\
\hline Residential, High Density & Log Home Prefabrication & Sand and Gravel Pits & Dog Racing Track \\
\hline High Density Residential Mobile & Plywood and Veneer Mill & Dolomite Quarry & Horse Racing Track \\
\hline Mobile Home Units, High Density & Pulp and Paper Mill & Inactive Strip Mine/Rock Quarry & Race Tracks \\
\hline Multiple DU Low Rise (<=2 Stories) & Saw Mill & Limerock Quarry & Marinas and Fish Camps \\
\hline Multiple DU High Rise ( $>=3$ Stories) & Timber Processing & Phosphate Mine & City Park \\
\hline High Density Residential Mixed & Wood Yard & Rock Quarries & Parks and Zoos \\
\hline Commercial and Services & Clays & Oil and Gas Fields & Zoo \\
\hline Commercial, Retail Sales and Serv. & Limerock Processing & Old Field & Community Recreational Facilities \\
\hline Shopping Center & Mineral Processing & Reclaimed land & Stadium \\
\hline Junk Yards & Phosphate Processing & Holding Ponds & Historic Sites \\
\hline Wholesale Sales and Services & Asphalt Plant & Institutional & Other Recreational \\
\hline Cultural and Entertainment & Oil and Gas Processing & Educational Facilities & Open Land \\
\hline Open Air Theater & Aircraft Building and Repair & Religious & Open Land (Urban) \\
\hline Campground & Boat Building and Repair & Military & Undeveloped Urban Land \\
\hline Motel & Container Manufacturer & National Guard Installation & Inactive Development Land \\
\hline Tourist Services & Electronics & Hospital & Urban Land in Transition \\
\hline Travel Trailer Park & Maintenance Yard & Medical and Health Care & Other Open Lands \\
\hline Liquified Gases & Mobile Home Manufacturer & Nursing Home & \\
\hline
\end{tabular}




\begin{tabular}{|l|l|l|l|}
\hline \multicolumn{3}{|c|}{ Agriculture } \\
\hline Improved Pasture & Fruit Orchard & Nurseries and Vineyards \\
\hline Unimproved Pasture & Peaches & Ornamental Nursery \\
\hline Woodland Pasture & Other Groves & Tree Nursery & Horse Farm \\
\hline Blueberries & Pecans & Sod Farm & Dairy \\
\hline Corn & Abandoned Tree Crops & Ornamentals & Kennel \\
\hline Row Crops & Feeding Operations & Vineyards & Other Open Lands (Rural) \\
\hline Field Crops & Cattle Feeding Operations & Floriculture & \\
\hline Tree Crops & Poultry Feeding Operation & Other Specialty Farm \\
\hline Citrus Groves & Swine Feeding Operations & Specialty Farms \\
\hline
\end{tabular}

\begin{tabular}{|l|l|l|l|}
\hline \multicolumn{3}{|c|}{ Rangeland } \\
\hline Herbaceous & Shrub and Brushland & Coastal Scrub & Mixed Rangeland \\
\hline Other Shrubs and Brush & Palmetto Prairie & & \\
\hline
\end{tabular}

\begin{tabular}{|l|l|l|l|}
\hline \multicolumn{3}{|c|}{ Upland Forest } \\
\hline Upland Coniferous Forests & Other Pine Upland Forests & Hardwood-Coniferous Mixed \\
\hline Pine Flatwoods & Upland Hardwood Forests & Dead Trees \\
\hline Longleaf Pine - Xeric Oaks & Oak - Pine - Hickory & Oak Scrub & Coniferous Plantations \\
\hline Longleaf Sandhill & Temperate Hardwood & Sand Pine Scrub \\
\hline Mesic Flatwoods & Beech - Magnolia & Sand Pines & Forest Regeneration Areas \\
\hline Pine - Mesic Oaks & Oak Sandhill & Australian Pine & \\
\hline
\end{tabular}

\begin{tabular}{|l|l|l|}
\hline \multicolumn{3}{|c|}{ Water } \\
\hline Streams and Waterways & Bays and Estuaries & Major Springs \\
\hline Lakes & Embayments Opening Directly into the Gulf & Slough Waters \\
\hline Reservoirs & Embayments not Opening Directly into the Gulf & Oceans, Seas, and Gulfs \\
\hline
\end{tabular}

\begin{tabular}{|l|l|l|l|}
\hline \multicolumn{3}{|c|}{ Wetlands } \\
\hline Wetland Hardwood Forests & Inland Ponds and Sloughs & Wetland Forested Mixed \\
\hline Bay Swamps & Mixed Wetland Hardwoods & Mixed Scrub-Shrub Wetland & Emergent Aquatic Vegetation \\
\hline Mangrove Swamps & Wetland Coniferous Forests & Shrub Swamp \\
\hline Gum Swamp & Cypress & Wetland Shrub & Non-Vegetated \\
\hline Titi Swamps & Pond Pine & Vegetated Non-forested Wetlands & Intermittent Ponds \\
\hline Bottomland Hardwood Swamp & Atlantic White Cedar & Freshwater Marshes \\
\hline River/Lake Swamp (Bottomland) & Cypress - Pine - Cabbage Palm & Saltwater Marshes \\
\hline
\end{tabular}




\begin{tabular}{|l|l|l|}
\hline \multicolumn{3}{|c|}{ Barren Land } \\
\hline Beaches & Disturbed Land & Borrow Areas \\
\hline Sand other than Beaches & Rural Land in Transition & Spoil Area \\
\hline
\end{tabular}

\begin{tabular}{|l|l|l|l|}
\hline \multicolumn{2}{|c|}{ Transportation, Communication, and Utilities } \\
\hline Transportation & Truck Terminal & Oil, Water, or Gas Lines \\
\hline Transportation Corridor & Divided Highway (Federal - State) & Auto Parking Facilities \\
\hline Airports & Highways & Highway Rest Area \\
\hline Commercial Airport & Limited Access Highway & Facilities under Construction \\
\hline General Aviation & Roads and Highways & Communications \\
\hline Private Airport & Two Lane Highway & Transmission Towers \\
\hline Railroads & Port Facilities & Communications Facilities & Electric Power Transmission Lines \\
\hline Bus Terminal & Canals and Locks & Utilities & Sater Supply Plant \\
\hline
\end{tabular}

